Research Article

\title{
Antiprolactinoma Effect of Hordenine by Inhibiting MAPK Signaling Pathway Activation in Rats
}

\author{
Xiong Wang $\mathbb{D}^{1},{ }^{1}$ Run-zhu Guo, ${ }^{2}$ Li Ma, ${ }^{1}$ Qiao-yan Ding, ${ }^{3}$ Jun-hua Meng, \\ Yong-gang Chen $\mathbb{D}^{1}{ }^{1}$ and Jin-hu $\mathrm{Wu} \mathbb{D}^{1}$ \\ ${ }^{1}$ Department of Pharmacy, Tongren Hospital Affiliated to Wuhan University (The Third Hospital of Wuhan), Wuhan, \\ Hubei, China \\ ${ }^{2}$ Department of Pharmacy, Wuhan Asia Heart Hospital, Wuhan, Hubei, China \\ ${ }^{3}$ College of Pharmacy, Hubei University of Chinese Medicine, Wuhan, Hubei, China
}

Correspondence should be addressed to Yong-gang Chen; 605333815@qq.com and Jin-hu Wu; wujinhuyx@163.com

Received 24 November 2019; Revised 20 February 2020; Accepted 9 March 2020; Published 23 April 2020

Academic Editor: Youn-Chul Kim

Copyright (C) 2020 Xiong Wang et al. This is an open access article distributed under the Creative Commons Attribution License, which permits unrestricted use, distribution, and reproduction in any medium, provided the original work is properly cited.

Prolactinomas are harmful to human health, and the clinical first-line treatment drug is bromocriptine. However, $20 \%$ prolactinomas patients did not respond to bromocriptine. Hordenine is an alkaloid separated from Fructus Hordei Germinatus, which showed significant antihyperprolactinemia activity in rats. The aim of this study was to explore the effect and mechanism of hordenine on prolactinomas in rats. The study used estradiol to induce prolactinomas, which caused the activation of the pituitary mitogen-activated protein kinase (MAPK) pathway in rats significantly. The treatment of hordenine restored estradiol, induced the overgrowth of pituitary gland, and reduced the prolactin (PRL) accumulation in the serum and pituitary gland of rats by blocking the MAPK (p38, ERK1/2, and JNK) activation and production of inflammatory cytokines, tumor necrosis factor- $\alpha$ (TNF- $\alpha$ ), interleukin- $\beta$ (IL-1 $\beta$ ), and interleukin-6 (IL-6). The antiprolactinoma effect of hordenine was mediated by inhibiting the MAPK signaling pathway activation in rats.

\section{Introduction}

As a benign adenomas, pituitary tumor accounts for $15 \%$ of all intracranial neoplasms $[1,2]$. The prevalence of pituitary tumor is relatively high in the general population, with approximately 77 cases per $100.000[3,4]$ and reached to a $20 \%$ prevalence in clinical occult pituitary adenomas [5]. In the clinic, prolactinomas are the most frequent pituitary tumors, which account for 50\% [6]. Prolactinomas are harmful to human body and usually causes severe symptoms such as hyperprolactinemia. The excessive PRL secretion by hyperprolactinemia often results in galactorrhea, decreased libido, and even infertility, especially in women. Although prolactinomas are benign, macroprolactinomas seem to be more resistant to drug therapy and aggressive than microprolactinomas [7]. At present, the pathogenesis theory of prolactinomas includes hypothalamic dysregulation and cancer gene mutation, but the exact pathogenesis is still unknown.

At present, the principle of treating prolactinomas is to decrease excessive serum PRL levels and to reduce tumor size, at last to restore pituitary function in patients. Under physiological conditions, prolactin production of the pituitary gland is inhibited by dopamine, which mainly acts on the dopamine D2 receptors (Drd2) expressed on the membranes of lactotroph cells [8]. In the clinic, most of prolactinomas patients show an effective response to the dopamine agonists (DAs) therapy such as bromocriptine and cabergoline, which are the first-line therapies compared with the surgical therapy [9]. However, $25 \%$ of prolactinoma patients do not respond to DA therapy, even at high doses of DA [10]. Furthermore, cabergoline is not on sale in China and other countries at present. Therefore, these problems bring a great challenge to the clinical treatments. 
As a classical signal molecule, the mitogen-activated protein kinase (MAPK) pathway plays important roles from extracellular signals to intracellular responses. In various diseases including cancers, the changes in MAPK signal transduction were found. More, the complex signaling cascades of MAPK were involved in tumorigenesis, development, and drug resistance [11]. The MAPK family includes many kinds of kinases, which often undergo changes and results in cancers. MAPKs include p38 MAPK, extracellular signal-regulated kinase (ERK1/2), and c-jun-Nterminal kinase (JNK1/2) [12]. In pathological conditions, extracellular stresses induce the production of cytokines and chemokines, resulting in the MAPK activation [13]. MAPK activation promoted the formation of inflammation and associated cancer [14]. Many studies have reported that p38 MAPK participated in the development and progress of lung cancer [15], prostate cancer [16-18], bladder cancer [19, 20], breast cancer [21, 22], liver cancer [23], transformed follicular lymphoma [24, 25], and leukemia [26]. Furthermore, p38 $\gamma$ showed a protumorigenic role for skin carcinogenesis [27]. And, p38 $\gamma$ regulated the oncogenic protein K-Ras of colorectal cancer [28]. Studies indicated that MAPK kinases can also inhibit tumor formation and progress [29]. MAPK are implicated in linking the inflammation and tumor development.

Hordenine is an alkaloid separated from traditional Chinese herb Fructus Hordei Germinatus. The structure of hordenine is shown in Figure 1. In previous study, we have reported that water extract and total alkaloids of Fructus Hordei Germinatus showed antihyperprolactinemia activity significantly [30, 31]. Furthermore, hordenine showed significant antihyperprolactinemia activity in rats [32]. This study was the first to study the possible mechanisms by which hordenine improved these abnormalities.

\section{Materials and Methods}

2.1. Materials. Bromocriptine was bought from Gedeon Richter Ltd. (Budapest, Hungary). Hordenine was bought from Chengdu Must Bio-Technology Co. Ltd. (Chengdu, PR China). The antibodies of prolactin (PRL), tumor necrosis factor- $\alpha$ (TNF- $\alpha$ ), interleukin- $1 \beta$ (IL-1 $\beta$ ), and interleukin-6 (IL-6) were obtained from Affinity Biosciences (Cincinnati, USA). The antibodies of nuclear factor kappa-B (NF- $\kappa \mathrm{B}$ ), p38, p-p38, JNK, p-JNK, ERK1/2, and p-ERK1/2 for rats were supported by Proteintech Group, Inc (Wuhan, PR China). The PRL enzyme-linked immunosorbent assay (ELISA) kit was bought from Wuhan Huamei Bioengineering Co., Ltd. (Wuhan, China).

2.2. Establishment of Prolactinoma Model Animals and Experimental Protocol. Eighty female F344 rats (weighing 250-300 g) were purchased from Beijing Wei Tong Li Hua Laboratory Animal Technology Co., Ltd. (Beijing, PR China). Rats were housed in a standard animal room at $22-25^{\circ} \mathrm{C}$ with a relative humidity of $60 \pm 5 \%$. And, they adapted to the environment of laboratory for one week before starting experiments.

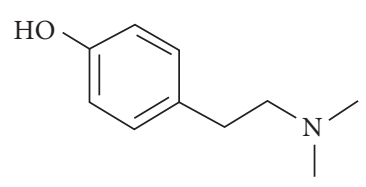

Figure 1: The structure of hordenine

Rats were randomly separated into seven groups: control group, sham-operated group (ovariectomized), model group (sham + estradiol injection), bromocriptine group (sham + estradiol injection $+0.393 \mathrm{mg} / \mathrm{kg}$ of bromocriptine, as positive control group), and hordenine (sham + estradiol injection $+38.2,76.4$ or $152.8 \mathrm{mg} / \mathrm{kg}$ of hordenine) groups $(n=10)$. Except control rats, other F344 rats were all ovariectomized. Except control group and sham-operated group rats, the abdominal cavity of rats was injected with $17 \alpha$-estradiol according to the dosage of $1 \mathrm{~mL}(2 \mathrm{mg})$ for every rat. It was carried out once every five days and lasted for 50 days. After 50-day injection of estradiol, hordenine was all dissolved in water and given to rats by means of intragastric administration daily at 9:00-10:00 a.m. for 30 days, respectively. The administration of drug dose was calculated according to the previous preclinical experiment data. Food and water intake was recorded daily.

\subsection{Nuclear Magnetic Resonance (NMR) Imaging Detection.} Appearance imaging of pituitary gland of rats was detected and recorded by animal NMR. And, the volume of pituitary gland in rats was calculated quantitatively by analytic software (Carimas 2.9).

2.4. Serum PRL Level Test. Blood was collected from the femoral artery of rats, and then the serum was separated immediately. For PRL ELISA, $2 \mu \mathrm{l}$ of serum was coated in a 96-well plate overnight at $4^{\circ} \mathrm{C}$. After being washed with $0.1 \%$ of Tween, samples were added with $10 \%$ of BSA solution, followed by avidin and biotin. The biotinylated hyaluronanbinding protein (Wuhan, Huamei) was then added into the samples and incubated for one hour at the room temperature. After the samples were washed for three times, avidinhorseradish peroxidase was added for incubation. 2,2-Azinobis (3-ethylbenzthiazoline-6-sulfonic acid) was used and set as a substrate, and the absorbance was set and read at $405 \mathrm{~nm}$.

2.5. Western Blot Analysis. After oral administration of drugs, rats were executed by carbon dioxide and the pituitary glands were excised, which were then immediately flashfrozen and stored at $-80^{\circ} \mathrm{C}$. Frozen tissue was weighed and then placed in lysis buffer ( $\mathrm{pH} 7.5$ of Tris, $150 \mathrm{mM}$ of $\mathrm{NaCl}$, and $1 \%$ of NP-40) with protease inhibitors to prepare the tissue protein lysates. Tissue homogenization was prepared with a homogenizer (Wuhan Servicebio). Sample proteins were separated on either 10 or $12 \%$ of SDS-PAGE gels. Gels were transferred to polyvinylidene fluoride membranes, and 5\% milk was added to block. Then, the membranes were incubated at $4^{\circ} \mathrm{C}$ overnight with primary antibodies: rabbit 
anti-rat PRL (Affinity Biosciences), rabbit anti-rat TNF- $\alpha$ (Affinity Biosciences), rabbit anti-rat IL-6 (Affinity Biosciences), rabbit anti-rat IL-1 $\beta$ (Affinity Biosciences), rabbit anti-rat NF- $\kappa$ B (Proteintech Group Inc.), rabbit anti-mouse p38 (Proteintech Group Inc.), rabbit anti-mouse p-p38 (Proteintech Group Inc.), rabbit anti-mouse ERK1/2 (Proteintech Group Inc.), rabbit anti-mouse p-ERK1/2 (Proteintech Group Inc.), rabbit anti-mouse JNK (Proteintech Group Inc.), rabbit anti-mouse p-JNK (Proteintech Group Inc.), and mouse anti- $\beta$-actin (Abclonal Biotech Co.). Blots were washed with TBST solution for three times, every ten minutes, and then secondary antibodies were added for incubation and imaged using ECL detection reagents (Shanghai Jiapeng). The protein expression was quantified using the Image J (Shanghai Jiapeng) and Quantity One software. The signal intensity of the bands was normalized to that of the corresponding $\beta$-actin band.

2.6. Statistical Analysis. All data were expressed as mean \pm SD. Statistical analyses were performed using GraphPad Instat software. The results were analyzed for statistical variance using an unpaired $t$-test or one-way ANOVA as appropriate. Results were considered statistically significant at $P<0.05$.

\section{Results}

3.1. Hordenine Inhibited the Overgrowth of Pituitary Gland in Rats. Nuclear magnetic resonance imaging detection showed that, compared with the control and sham-operated control rats, estradiol increased the pituitary gland volume in rats significantly $(P<0.01)$. Hordenine succeeded in restoring the overgrowth of pituitary gland at $152.8(P<0.01), 76.4$ $(P<0.01)$, and $38.2 \mathrm{mg} / \mathrm{kg}(P<0.05)$ in this model. Bromocriptine at $0.393 \mathrm{mg} / \mathrm{kg}$ significantly inhibited the overgrowth of pituitary gland in rats $(P<0.05)$ (Figures 2 and 3 ).

3.2. Hordenine Inhibited the Serum Prolactin Level of Rats. Compared with the control and sham-operated control rats, estradiol significantly increased the serum prolactin level in rats $(P<0.01)$. Hordenine restored the increase of serum prolactin level at $152.8(P<0.05), 76.4(P<0.01)$, and $38.2 \mathrm{mg} / \mathrm{kg} \quad(P<0.05)$ in this model. Bromocriptine at $0.393 \mathrm{mg} / \mathrm{kg}$ significantly lowered the serum prolactin level in rats $(P<0.01)$ (Figure 4$)$.

3.3. Hordenine Inhibited Prolactin Overproduction in Estradiol-Induced Rats through Regulating MAPK Signaling Pathway. Compared with the control and sham-operated control rats, estradiol significantly increased pituitary gland protein level of PRL $(P<0.01)$, p-p38 $(P<0.01)$, p-ERK1/2 $(P<0.01), \quad$ p-JNK $(P<0.01), \quad$ NF- $\kappa \mathrm{B} \quad(P<0.01), \quad$ TNF- $\alpha$ $(P<0.01)$, IL-1 $\beta \quad(P<0.01)$, and IL-6 $(P<0.01)$ in rats. Hordenine at $38.2 \mathrm{mg} / \mathrm{kg}$ significantly downregulated the protein level of the pituitary gland PRL $(P<0.01)$, p-p38 $(P<0.01)$, p-ERK1/2 $(P<0.01)$, p-JNK $(P<0.01), \mathrm{NF}-\kappa \mathrm{B}$ $(P<0.01)$, TNF- $\alpha \quad(P<0.01)$, IL-1 $\beta \quad(P<0.01)$, and IL-6
$(P<0.01)$, at $76.4 \mathrm{mg} / \mathrm{kg}$ lowered pituitary gland protein level of PRL $(P<0.01)$, p-ERK1/2 $(P<0.01)$, p-JNK $(P<0.01)$, TNF- $\alpha(P<0.01)$, IL-1 $\beta(P<0.01)$, and IL-6 $(P<0.01)$, and at $152.8 \mathrm{mg} / \mathrm{kg}$ only lowered p-ERK1/2 $(P<0.01), \quad$ TNF- $\alpha$ $(P<0.01)$, and IL-1 $\beta(P<0.01)$ in estradiol-induced rats. And, bromocriptine also succeeded in restoring estradiolinduced overexpression of pituitary gland protein level of p-p38 $(P<0.05)$, p-ERK1/2 $(P<0.01)$, and IL-1 $\beta(P<0.01)$ at $0.393 \mathrm{mg} / \mathrm{kg}$ in this model (Figures 5 and 6).

\section{Discussion}

As one of the complementary and alternative medicine options, traditional Chinese herbal drug is currently commonly used and popular in China. Fructus Hordei Germinatus has been used to cure hyperprolactinemia for thousands of years. Hordenine, an alkaloid and a major constituent of Fructus Hordei Germinatus, has been previously demonstrated to possess antihyperprolactinemia effects [32]. It attracted our attention for further development. Thus, this study investigated the effect and mechanism of hordenine on prolactinomas induced by estradiol in rats. We used F344 rats to prepare the model and demonstrated that hordenine restored the overgrowth of pituitary gland and reduced the PRL accumulation in the serum and pituitary gland of rats. The results confirmed that hordenine may improve the abnormalities of prolactinomas.

We then studied the possible anti-prolactinomas mechanism of hordenine in rats. MAPK is an important transmitter of signal from cell surface to nucleus, which is followed by phosphorylation of many kinds of cytosolic proteins associated with cell proliferation, cell invasion, cell differentiation, cell migration, and cell apoptosis [33]. MAPK is composed of p38 MAPK, c-jun-N-terminal kinase (JNK1/2), and extracellular signal-regulated kinase (ERK1/ 2) [12]. The p38 MAPK is stimulated and activated by various kinds of cellular stresses, such as lipopolysaccharides (LPS), ultraviolet light, inflammatory cytokines, and growth factors and then makes this signaling pathway a potential therapeutic target for inflammatory diseases [34]. The p38 MAPK have tumorigenic functions in certain contexts [35]. We used western blotting to analyze the effects of hordenine on MAPK protein expressions in estradiol-induced prolactinomas rats. The western blotting results showed that estradiol significantly increased pituitary gland protein levels of PRL, p-p38, p-ERK1/2, p-JNK, NF- $\kappa$ B, TNF- $\alpha$, IL- $1 \beta$, and IL-6 in rats, which were reversed by hordenine treatment. Our results suggested that the the MAPK pathway and cytokines/ILs were significantly elevated in the progress of prolactinoma model, and hordenine inhibited the production of PRL by regulating MAPKs signaling. However, the moleclular mechanism of the MAPK pathway and cytokines/ILs in the prolactinomas model is not very clear and is being studied by our group.

At present, the clinical first-line treatment drugs for prolactinomas are bromocriptine and cabergoline, which are dopamine D2 receptor agonists. However, 25\% of patients do not respond to this therapy, and there are obvious side effects such as nausea. Thus, it is necessary to study new 


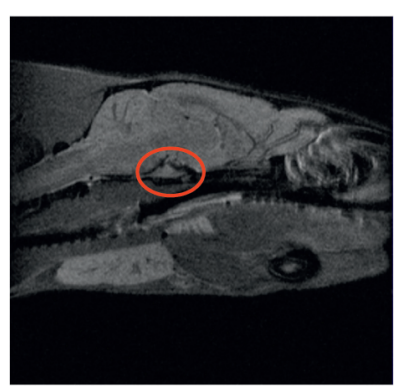

(a)

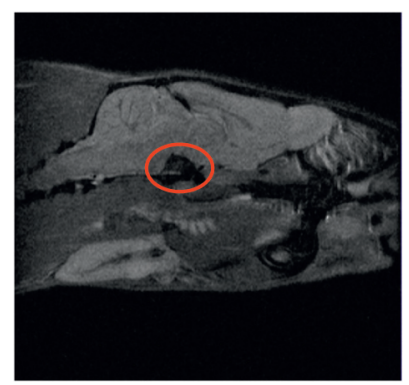

(b)

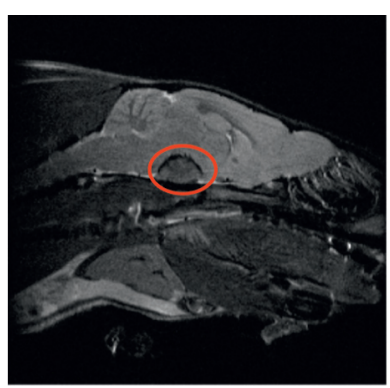

(c)

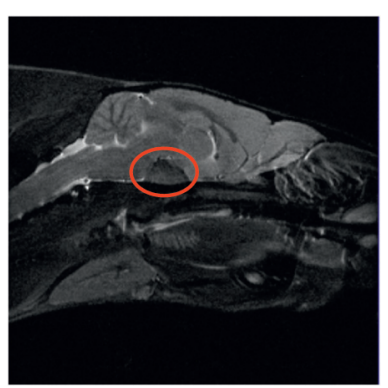

(d)

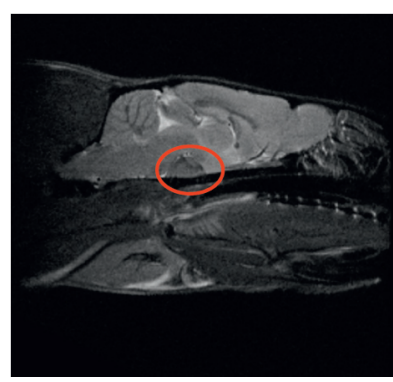

(e)

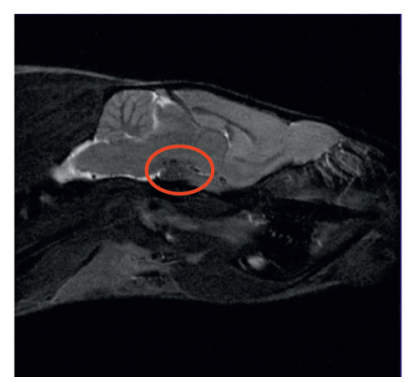

(f)

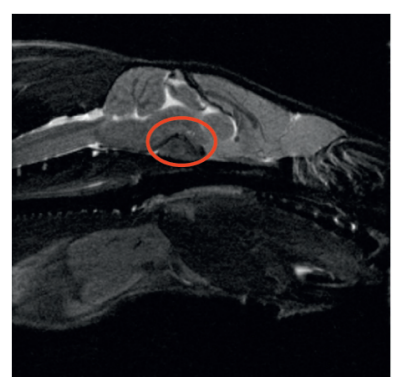

(g)

Figure 2: Pituitary gland imaging of rats by nuclear magnetic resonance: (a) control, (b) sham-operated control, (c) model control, (d) model + bromocriptine, (e) model + hordenine (152.8 mg/kg), (f) model + hordenine (76.4 mg/kg), and (g) model + hordenine (38.2 mg/kg).

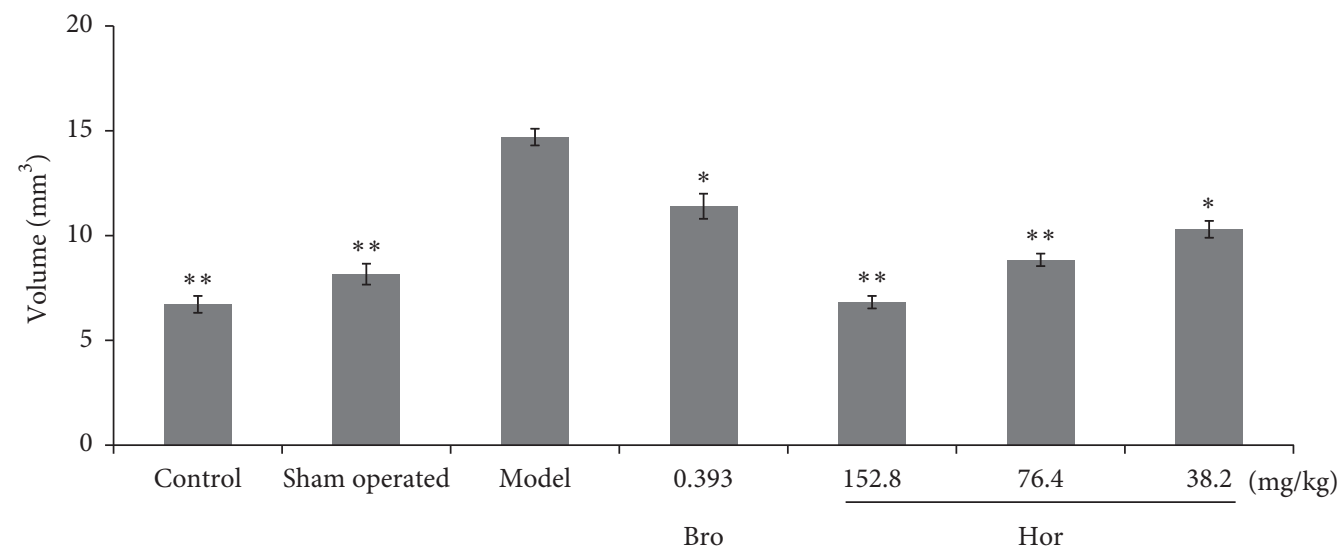

FIGURE 3: Pituitary gland volume of rats by nuclear magnetic resonance. Bro: bromocriptine; Hor: hordenine. Data were shown as mean $\pm \mathrm{SD}(n=10) .{ }^{* *} P<0.01,{ }^{*} P<0.05$ compared with the model group.

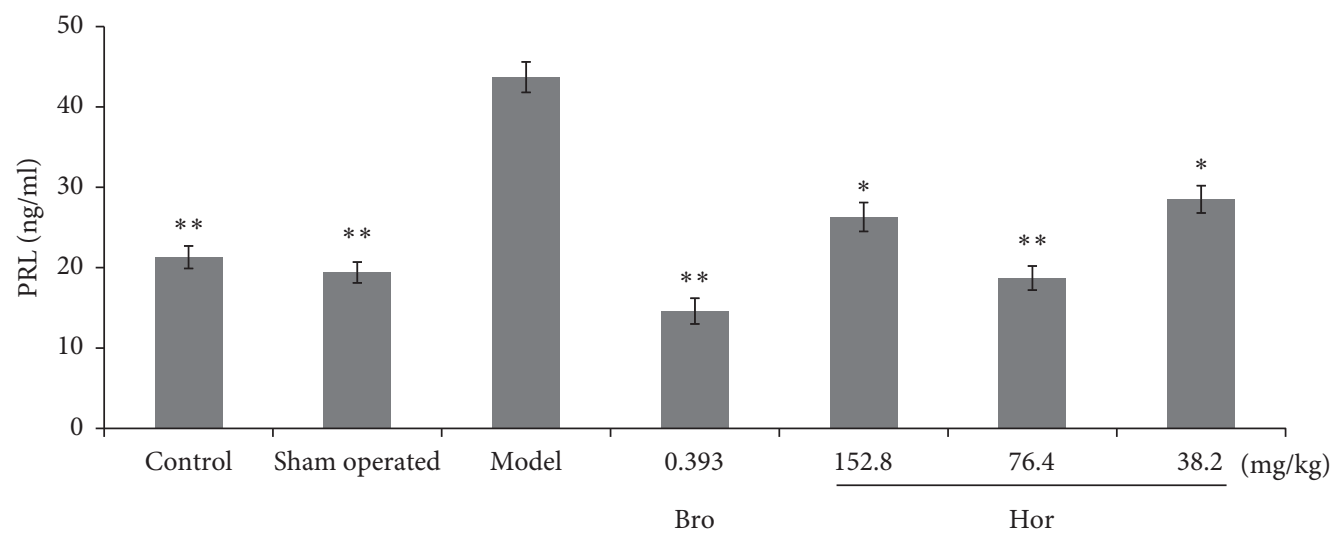

Figure 4: Serum prolactin level of rats by ELISA. Bro: bromocriptine; Hor: hordenine. Data were shown as mean $\pm \operatorname{SD}(n=8)$. ${ }^{* *} P<0.01$, ${ }^{*} P<0.05$ compared with the model group. 


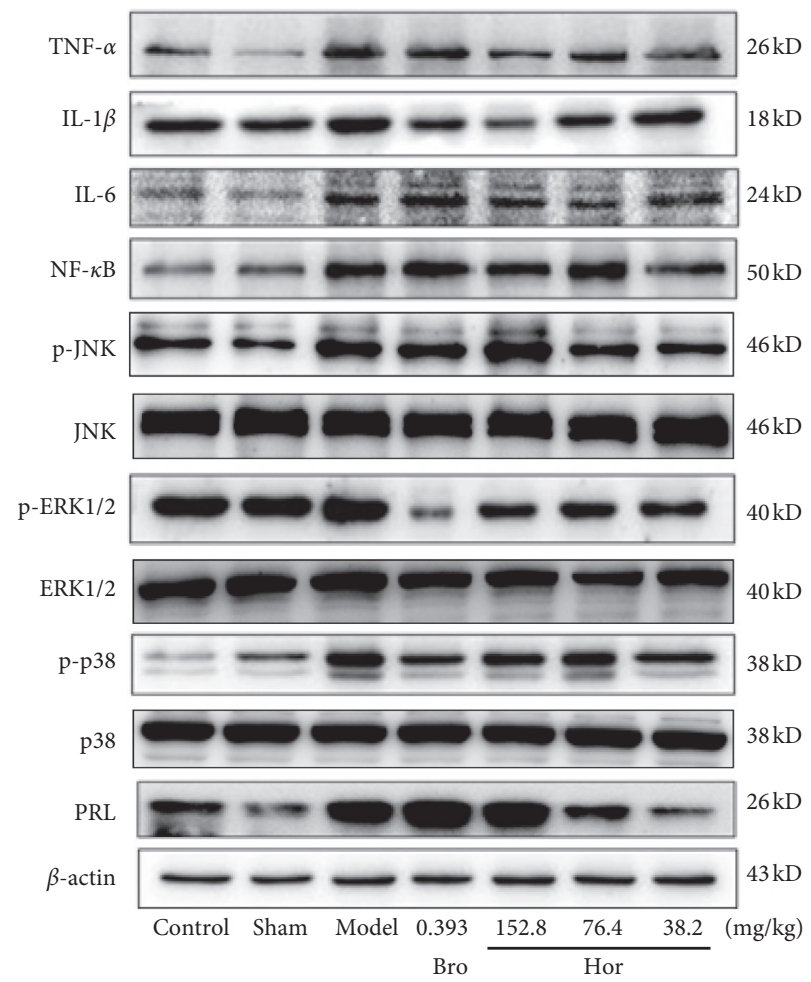

FIGURE 5: Western blot image of pituitary gland protein expressions in rats. Control: control group, Sham: sham-operated control group, Model: model control group, Bro: bromocriptine group, Hor: hordenine group.

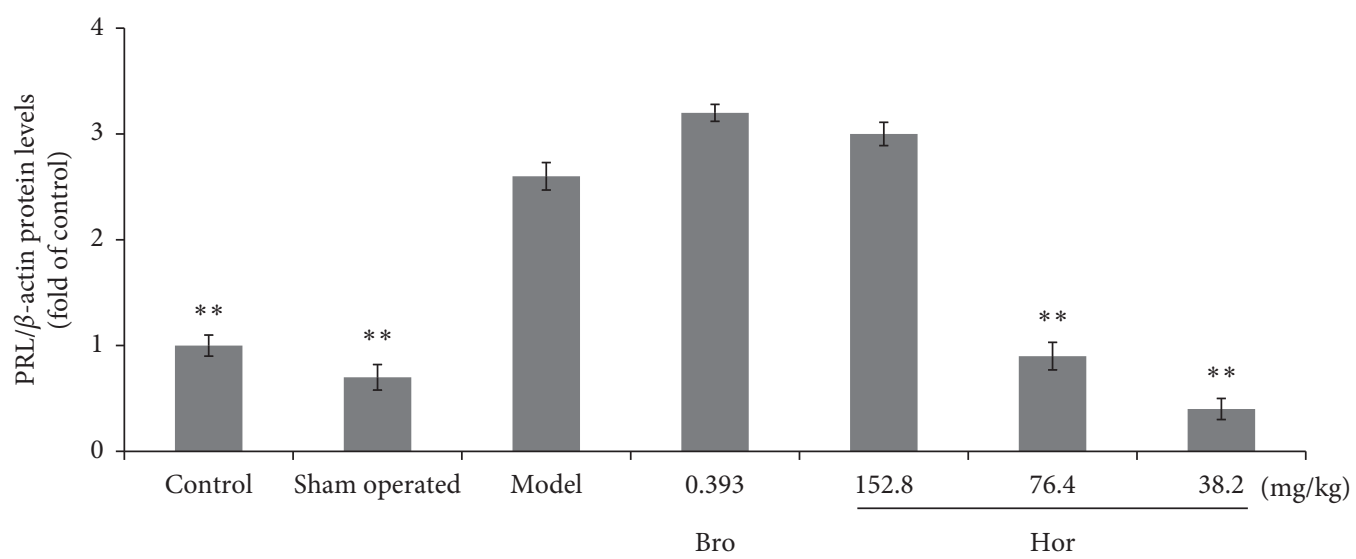

(a)

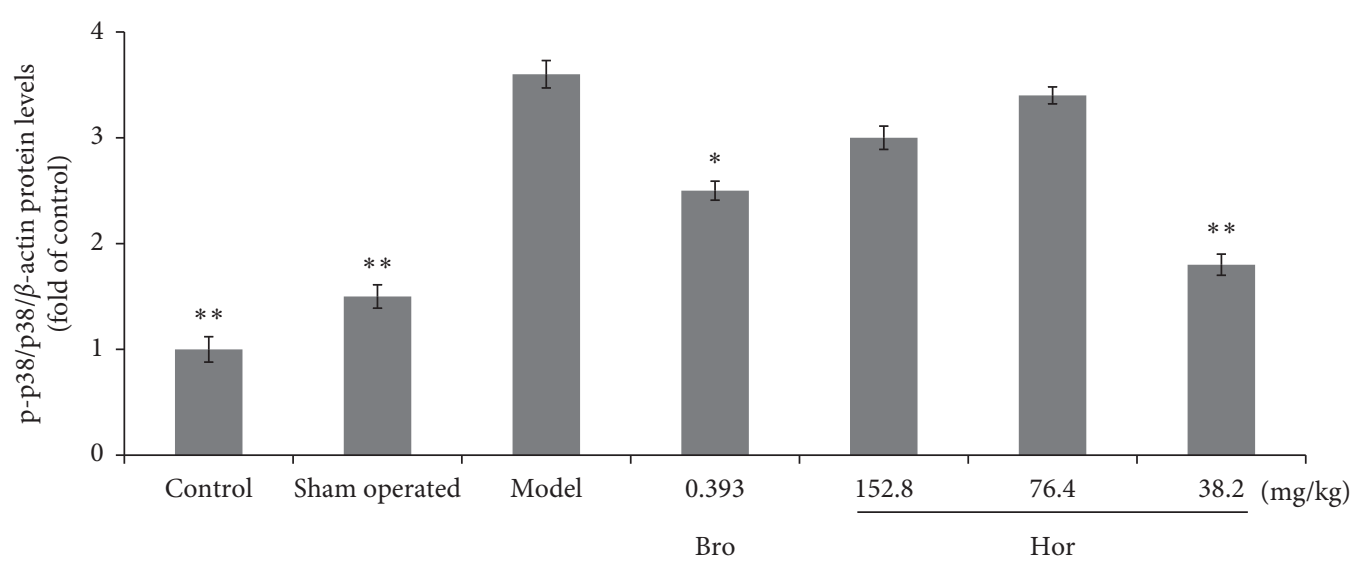

(b)

Figure 6: Continued. 


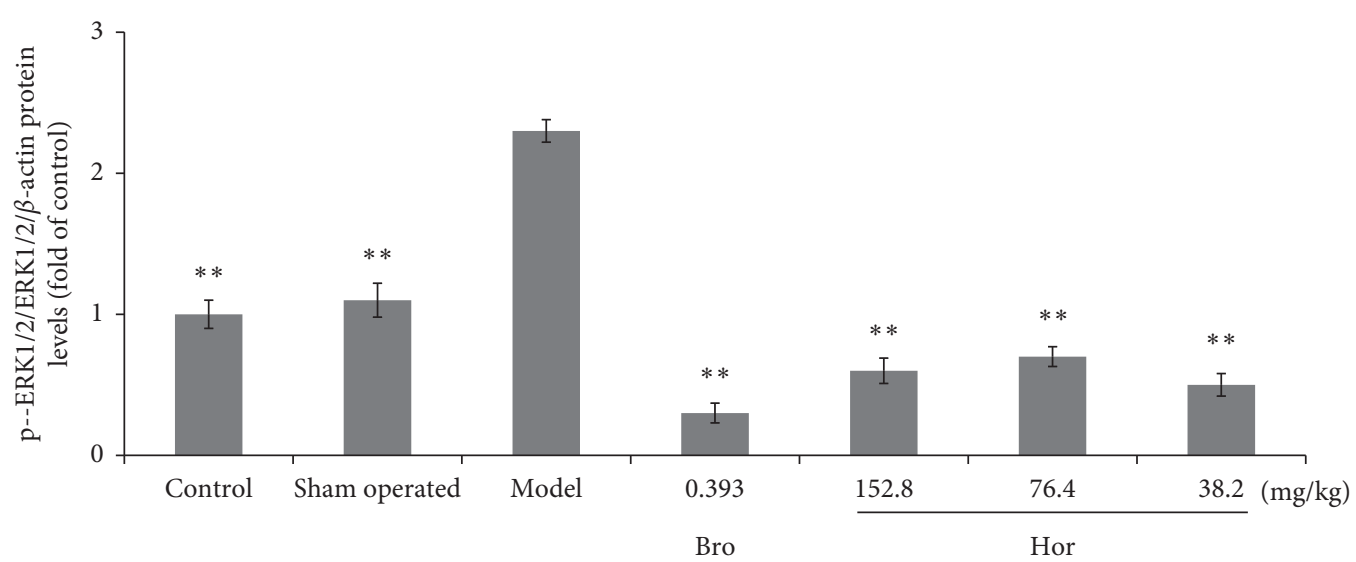

(c)

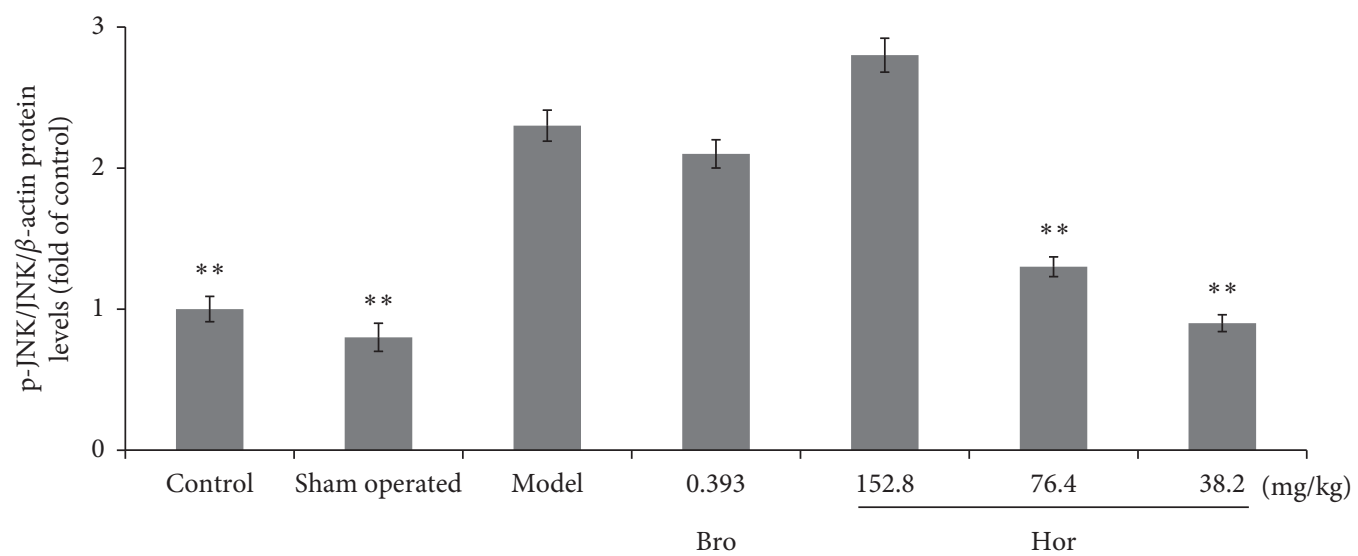

(d)

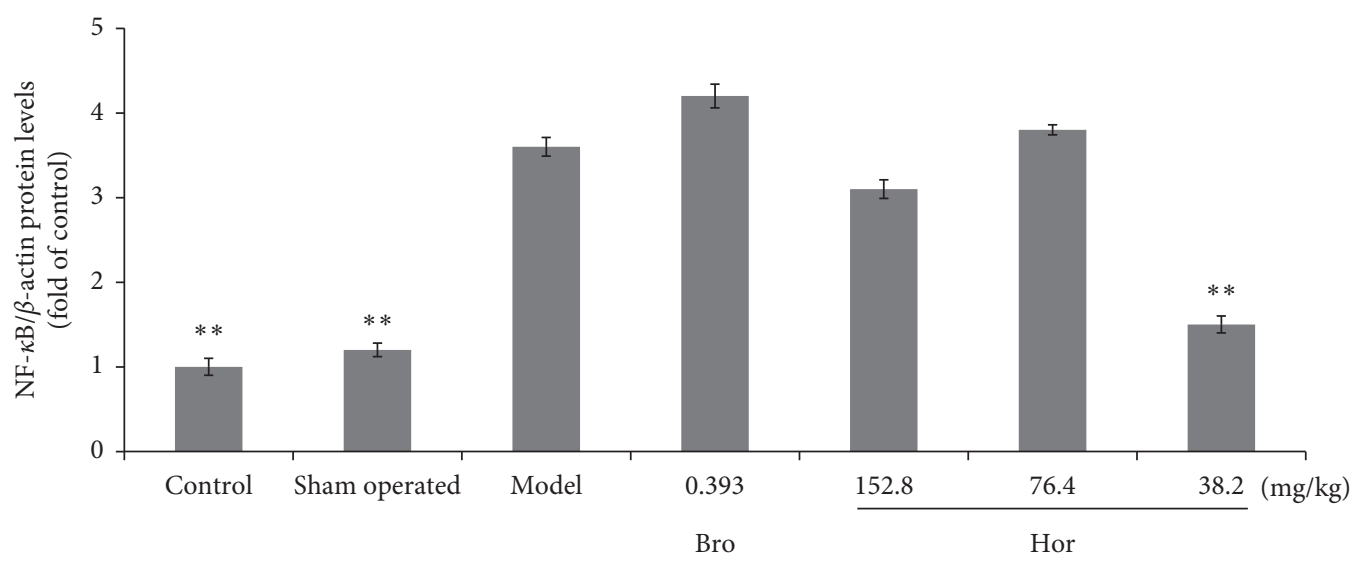

(e)

Figure 6: Continued. 


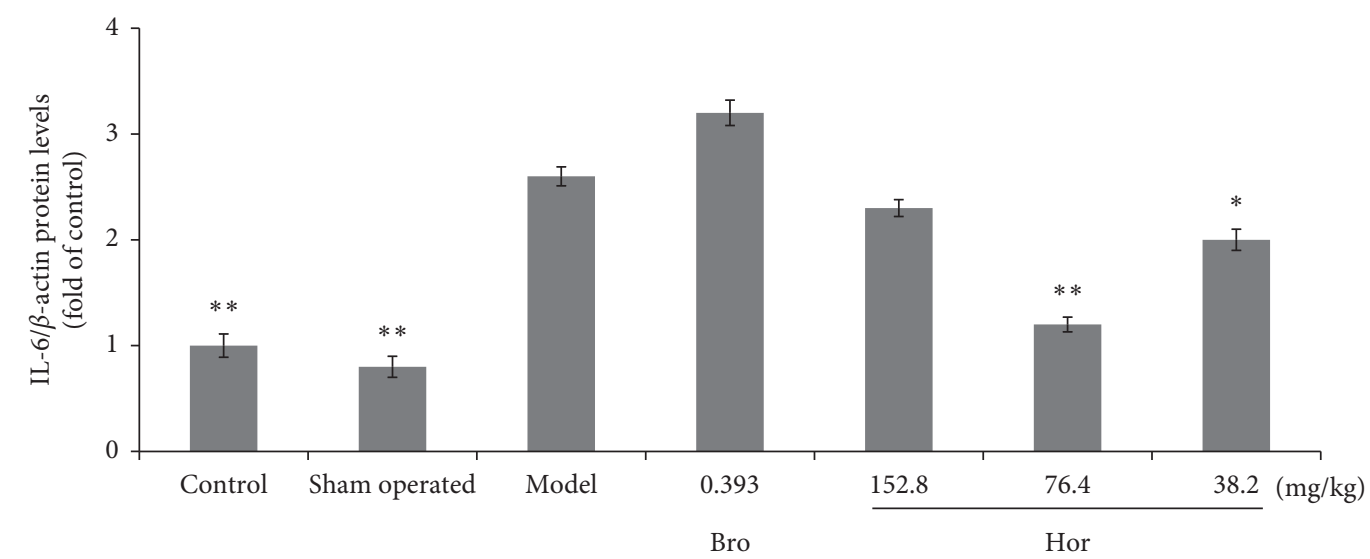

(f)

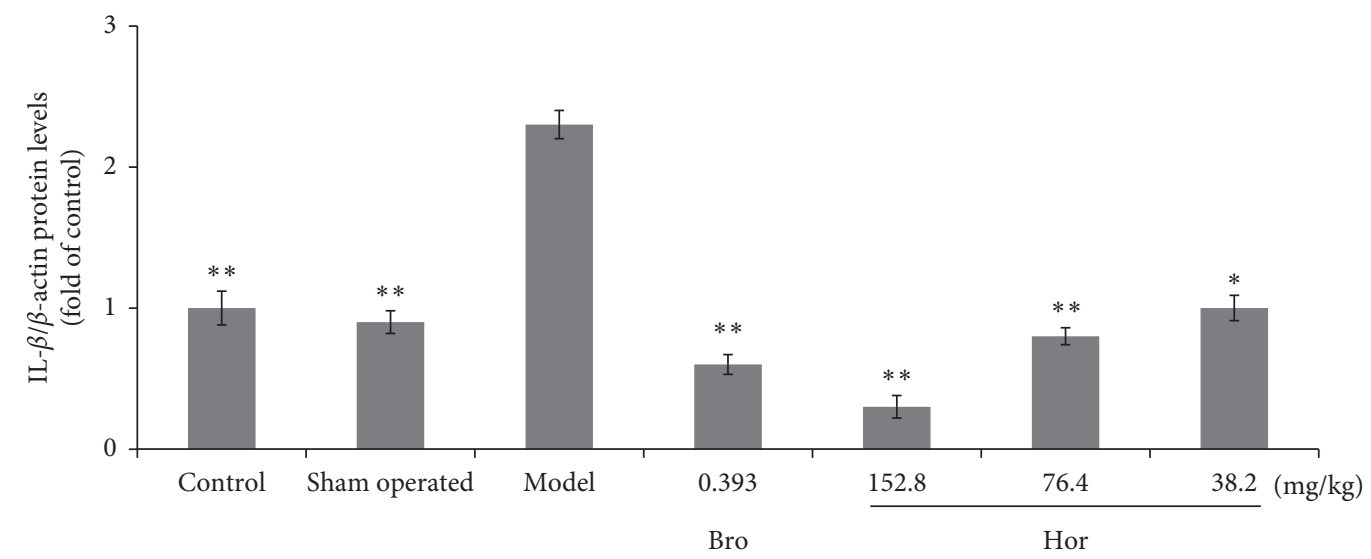

(g)

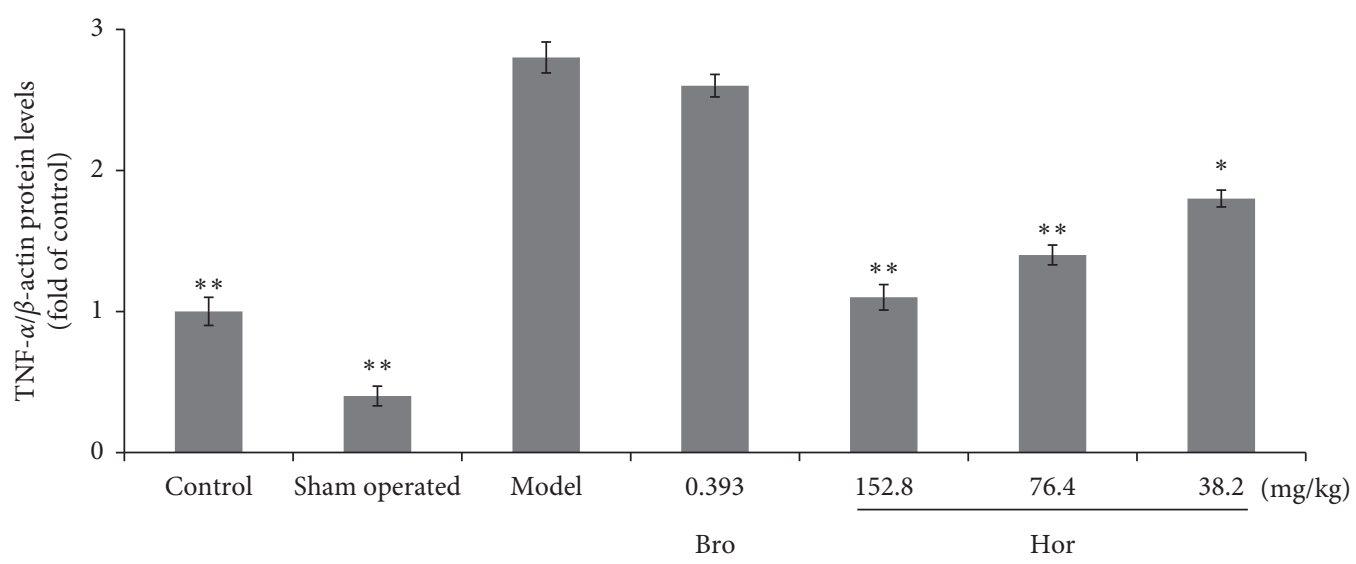

(h)

Figure 6: Protein levels of pituitary PRL (a), p-p38/p38 (b), p-ERK1/2/ERK1/2 (c), p-JNK/JNK (d), NF- $\kappa$ B (e), IL-6 (f), IL-1 $\beta$ (g), and TNF$\alpha$ (h) of rats were measured by western blot. Data were shown as mean $\pm \operatorname{SD}(n=4)$. Compared with the model group, ${ }^{* *} P<0.01,{ }^{*} P<0.05$.

targets and drugs for the treatment of prolactinomas. The present study showed that 30 days of treatment of hordenine inhibited the overgrowth of pituitary glands, reduced the prolactin expression in the serum and pituitary gland, and decreased the expression of p-p38, p-ERK1/2, p-JNK, NF$\kappa \mathrm{B}, \mathrm{TNF}-\alpha$, IL- $1 \beta$, and IL- 6 of pituitary glands in rats. Based on the findings of this study, it suggests that there is a regulatory effect of hordenine on MAPK signaling. We supposed that the antiprolactinoma-like effects of hordenine are mediated by inhibiting the MAPK signaling activation in estradiol-induced rats.

\section{Conclusion}

In conclusion, the present study showed that hordenine (76.4 and $152.8 \mathrm{mg} / \mathrm{kg}$ ) inhibited the overgrowth and PRL 
expression of the pituitary gland in estradiol-induced rats via regulation of the MAPK pathway, lowering the p-p38, $\mathrm{p}-\mathrm{ERK} 1 / 2$, and $\mathrm{p}$-JNK protein expression and the production of inflammatory cytokines TNF- $\alpha$, IL- $1 \beta$, and IL-6, which provided the new therapeutic targets and drugs for treating prolactinomas.

\section{Data Availability}

The data used to support the findings of this study are included within the article.

\section{Disclosure}

Yong-gang Chen and Jin-hu $\mathrm{Wu}$ are co-corresponding authors.

\section{Conflicts of Interest}

The authors declare that there are no conflicts of interest.

\section{Authors' Contributions}

Yong-gang Chen and Jin-hu Wu contributed equally to this work.

\section{Acknowledgments}

Financial support for this work was provided by grants from the Natural Science Foundation of China (No. 81603352), Hubei Natural Science Foundation (2017CFB125), and Scientific Research Project Funds for Wuhan Health and Family Planning Commission (WZ16A10).

\section{References}

[1] W. E. Farrell, "Pituitary tumours: findings from whole genome analyses," Endocrine-Related Cancer, vol. 13, pp. 707716, 2006.

[2] S. Melmed, "Pituitary tumors," Endocrinology and Metabolism Clinics of North America, vol. 44, no. 1, pp. 1-9, 2015.

[3] A. F. Daly, M. A. Tichomirowa, and A. Beckers, "The epidemiology and genetics of pituitary adenomas," Best Practice \& Research Clinical Endocrinology \& Metabolism, vol. 23, no. 5, pp. 543-554, 2009.

[4] A. Fernandez, N. Karavitaki, and J. A. H. Wass, "Prevalence of pituitary adenomas: a community-based, cross-sectional study in Banbury," Clinical Endocrinology, vol. 72, no. 3, pp. 377-382, 2010.

[5] S. Ezzat, S. L. Asa, W. T. Couldwell et al., "The prevalence of pituitary adenomas," Cancer, vol. 101, no. 3, pp. 613-619, 2004.

[6] A. Ciccarelli, A. F. Daly, and A. Beckers, "The epidemiology of prolactinomas," Pituitary, vol. 8, no. 1, pp. 3-6, 2005.

[7] A. Wong, J. A. Eloy, W. T. Couldwell, and J. K. Liu, "Update on prolactinomas. Part 1: clinical manifestations and diagnostic challenges," Journal of Clinical Neuroscience, vol. 22, no. 10, pp. 1562-1567, 2015.

[8] N. Ben-Jonathan and R. Hnasko, "Dopamine as a prolactin (PRL) inhibitor," Endocrine Reviews, vol. 22, no. 6, pp. 724763, 2001.
[9] A. Wong, J. A. Eloy, W. T. Couldwell, and J. K. Liu, "Update on prolactinomas. Part 2: treatment and management strategies," Journal of Clinical Neuroscience, vol. 22, no. 10, pp. 1568-1574, 2015.

[10] L. Vroonen, M.-L. Jaffrain-Rea, P. Petrossians et al., "Prolactinomas resistant to standard doses of cabergoline: a multicenter study of 92 patients," European Journal of Endocrinology, vol. 167, no. 5, pp. 651-662, 2012.

[11] M. Burotto, V. L. Chiou, J.-M. Lee, and E. C. Kohn, "The MAPK pathway across different malignancies: a new perspective," Cancer, vol. 120, no. 22, pp. 3446-3456, 2014.

[12] R. Seger and E. G. Krebs, "The MAPK signaling cascade," The FASEB Journal, vol. 9, no. 9, pp. 726-735, 1995.

[13] A. Plotnikov, E. Zehorai, S. Procaccia, and R. Seger, "The MAPK cascades: signaling components, nuclear roles and mechanisms of nuclear translocation," Biochimica et Biophysica Acta (BBA)-Molecular Cell Research, vol. 1813, no. 9, pp. 1619-1633, 2011.

[14] M. P. Smith, B. Sanchez-Laorden, K. O’Brien et al., "The immune microenvironment confers resistance to MAPK pathway inhibitors through macrophage-derived TNF," Cancer Discovery, vol. 4, no. 10, pp. 1214-1229, 2014.

[15] A. K. Greenberg, S. Basu, J. Hu et al., "Selective p38 activation in human non-small cell lung cancer," American Journal of Respiratory Cell and Molecular Biology, vol. 26, no. 5, pp. 558-564, 2002.

[16] J.-I. Park, M.-G. Lee, K. Cho et al., "Transforming growth factor- $\beta 1$ activates interleukin- 6 expression in prostate cancer cells through the synergistic collaboration of the Smad2, p38$\mathrm{NF}-\kappa \mathrm{B}, \mathrm{JNK}$, and Ras signaling pathways," Oncogene, vol. 22, no. 28, pp. 4314-4332, 2003.

[17] L. Khandrika, R. Lieberman, S. Koul et al., "Hypoxia-associated p38 mitogen-activated protein kinase-mediated androgen receptor activation and increased HIF- $1 \alpha$ levels contribute to emergence of an aggressive phenotype in prostate cancer," Oncogene, vol. 28, no. 9, pp. 1248-1260, 2009.

[18] P. D. Maroni, S. Koul, R. B. Meacham, and H. K. Koul, "Mitogen activated protein kinase signal transduction pathways in the prostate," Cell Commun Signal, vol. 2, no. 5, 2004.

[19] B. Kumar, J. Sinclair, L. Khandrika, S. Koul, S. Wilson, and H. K. Koul, "Differential effects of MAPKs signaling on the growth of invasive bladder cancer cells," International Journal of Oncology, vol. 34, no. 6, pp. 1557-1564, 2009.

[20] B. Kumar, S. Koul, J. Petersen et al., "p38 mitogen-activated protein kinase-driven MAPKAPK2 regulates invasion of bladder cancer by modulation of MMP-2 and MMP-9 activity," Cancer Research, vol. 70, no. 2, pp. 832-841, 2010.

[21] P.-W. Tsai, S.-G. Shiah, M.-T. Lin, C.-W. Wu, and M.-L. Kuo, "Up-regulation of vascular endothelial growth factor $\mathrm{C}$ in breast cancer cells by heregulin- $\beta 1$," Journal of Biological Chemistry, vol. 278, no. 8, pp. 5750-5759, 2003.

[22] C. Suarez-Cuervo, M. A. Merrell, L. Watson et al., "Breast cancer cells with inhibition of p38," Clinical \& Experimental Metastasis, vol. 21, no. 6, pp. 525-533, 2004.

[23] K. Iyoda, Y. Sasaki, M. Horimoto et al., "Involvement of the p38 mitogen-activated protein kinase cascade in hepatocellular carcinoma," Cancer, vol. 97, no. 12, pp. 3017-3026, 2003.

[24] K. S. J. Elenitoba-Johnson, S. D. Jenson, R. T. Abbott et al., "Involvement of multiple signaling pathways in follicular lymphoma transformation: p38-mitogen-activated protein kinase as a target for therapy," Proceedings of the National Academy of Sciences, vol. 100, no. 12, pp. 7259-7264, 2003. 
[25] Z. Lin, D. K. Crockett, S. D. Jenson, M. S. Lim, and K. S. J. Elenitoba-Johnson, "Quantitative proteomic and transcriptional analysis of the response to the p38 mitogenactivated protein kinase inhibitor SB203580 in transformed follicular lymphoma cells," Molecular \& Cellular Proteomics, vol. 3, no. 8, pp. 820-833, 2004.

[26] R. Y. Liu, C. Fan, G. Liu, N. E. Olashaw, and K. S. Zuckerman, "Activation of p38 mitogen-activated protein kinase is required for tumor necrosis factor- $\alpha$-supported proliferation of leukemia and lymphoma cell lines," Journal of Biological Chemistry, vol. 275, no. 28, pp. 21086-21093, 2000.

[27] E. M. Schindler, A. Hindes, E. L. Gribben et al., "p38 mitogenactivated protein kinase is essential for skin tumor development in mice," Cancer Research, vol. 69, no. 11, pp. 46484655, 2009.

[28] X. Qi, N. M. Pohl, M. Loesch et al., "p38 $\alpha$ antagonizes p38 $\gamma$ activity through c-Jun-dependent ubiquitin-proteasome pathways in regulating ras transformation and stress response," Journal of Biological Chemistry, vol. 282, no. 43, pp. 31398-31408, 2007.

[29] M. I. Cerezo-Guisado, P. d. Reino, G. Remy et al., "Evidence of p38 $\gamma$ and p38 involvement in cell transformation processes," Carcinogenesis, vol. 32, no. 7, pp. 1093-1099, 2011.

[30] X. Wang, L. Ma, E. J. Zhang et al., "Water extract of Fructus Hordei Germinatus shows antihyperprolactinemia activity via dopamine D2 receptor," Evidence-Based Complementary and Alternative Medicine, vol. 2014, Article ID 579054, 7 pages, 2014.

[31] W. Xiong, M. Li, and W. Jin-Hu, “Therapeutic effects of total alkaloids of Fructus Hordei Germinatus in hyperprolactinemis rats," Pakistan Journal of Pharmaceutical Sciences, vol. 27, pp. 2087-2093, 2014.

[32] R. Z. Guo, X. Wang, L. Ma, Y. G. Chen, and J. H. Wu, "Inhibition effects of hordenine on prolactin secretion in hyperprolactinemia rats," Chinese Traditional Patent Medicine, vol. 40, pp. 2386-2389, 2018.

[33] S. Setia, B. Nehru, and S. N. Sanyal, "Upregulation of MAPK/ Erk and PI3K/Akt pathways in ulcerative colitis-associated colon cancer," Biomedicine \& Pharmacotherapy, vol. 68, no. 8, pp. 1023-1029, 2014.

[34] Y.-C. Hsiao, W.-H. Kuo, P.-N. Chen et al., "Flavanone and 2' $\mathrm{OH}$ flavanone inhibit metastasis of lung cancer cells via downregulation of proteinases activities and MAPK pathway," Chemico-Biological Interactions, vol. 167, no. 3, pp. 193-206, 2007.

[35] A. Risco and A. Cuenda, "New insights into the p38gamma and p38delta MAPK pathways," Journal of Signal Transduction, vol. 2012, Article ID 520289, 8 pages, 2012. 